\title{
Ipsilateral Shoulder and Elbow Dislocation: A Case Report
}

\author{
S Meena, MS Orth, P Saini, MS Orth, G Rustagi*, MS Orth, G Sharma, MS Orth \\ Department of Orthopaedics, All India Institute of Medical Sciences (AlIMS), \\ New Delhi, India \\ *Department of Orthopaedics, Maulana Azad Medical College, New Delhi, India
}

\begin{abstract}
Simultaneous ipsilateral shoulder and elbow joint dislocation is a rare injury. In most reported cases, shoulder joint dislocation was missed and diagnosed at a later time. We present the case of a 30 -year-old male who dislocated his ipsilateral shoulder and elbow joint following a motor vehicle accident. Keeping a high index of suspicion, radiographs of both joints were obtained. Under general anaesthesia, the elbow joint was reduced before the shoulder joint. In the final follow up after 1.5 years, the patient had returned to his occupation and enjoyed a full range of motion. The purpose of this report is to describe a very rare injury and to emphasise the importance of maintaining a high index of suspicion in such cases.
\end{abstract}

Key Words:

Dislocation, shoulder, elbow, ipsilateral

\section{INTRODUCTION}

The shoulder joint is the most commonly dislocated joint in the body. The elbow is the second most commonly dislocated joint after the shoulder. Ipsilateral shoulder and elbow dislocation is a very rare injury and only a few cases have been reported in the literature ${ }^{1-5}$. In most reported cases, shoulder joint dislocation was missed and diagnosed later. We report the case of a 30-year-old man, who suffered anterior shoulder dislocation and posterior elbow dislocation in the same limb following high velocity trauma and was treated conservatively with good results.

\section{CASE REPORT}

A 30-year-old male was brought to the emergency room by his friends after he sustained a motor vehicle accident. The patient was driving and there was head-on collision with another vehicle. On presentation he complained of severe pain over his right shoulder and right elbow joint. The affected extremity was supported with the opposite hand to keep the shoulder abducted, externally rotated and elbow flexed due to pain. On examination there was gross deformity of the elbow joint, contour of the shoulder joint was lost and its circumference increased. The humeral head was palpable anteriorly beneath the skin. Range of motion of both the shoulder and elbow joints were globally restricted and the patient was not allowing movement of his right upper limb due to pain. There was no other injury or any distal neurovascular deficit. Radiographs of both joints revealed anterior dislocation of shoulder and posterior dislocation of elbow without any associated fracture (Figures $1 \mathrm{a}, 1 \mathrm{~b}$ and 1c). Both joints were reduced urgently under general anaesthesia. The elbow was first reduced followed by reduction of shoulder joint by Kocher's method. Next, following assessment for stability the elbow joint was placed in a padded dressing with a posterior plaster splint in full supination at 90 degrees of flexion. For the shoulder joint, an immobilizer was applied. Post reduction radiographs revealed concentric reduction of the shoulder and elbow joints (Figures 2a, 2b and 2c).

After a period of three weeks, the elbow slab was removed and pendulum exercises of the shoulder were initiated with a sling in place. At 4 weeks post-procedure, the patient had gained flexion-extension of 50-120 degrees at the elbow joint and nearly full range of motion at the shoulder joint. At 6 month follow up patient had gained full range of motion of both the joints. Final follow up at 1.5 years showed full range of motion without any pain and the patient had returned to his previous occupation of taxi driver.

\section{DISCUSSION}

Simultaneous shoulder and elbow joint dislocation in the same limb is an extremely rare injury ${ }^{4}$. Suman first reported such a case in a 31-year-old man involved in a road traffic accident under the influence of alcohol ${ }^{2}$. To date only seven cases have been reported in the english literature so far. In some case reports, shoulder dislocation was initially missed and was subsequently detected in the follow up ${ }^{3-4}$. Ali et al. stressed the importance of careful examination and maintaining a high degree of suspicion following trauma to the humerus and elbow as an ipsilateral shoulder dislocation can be easily missed, especially in obese patients when changes of the shoulder contour can be minimal 3. Those patients who have associated head injury or are under the 


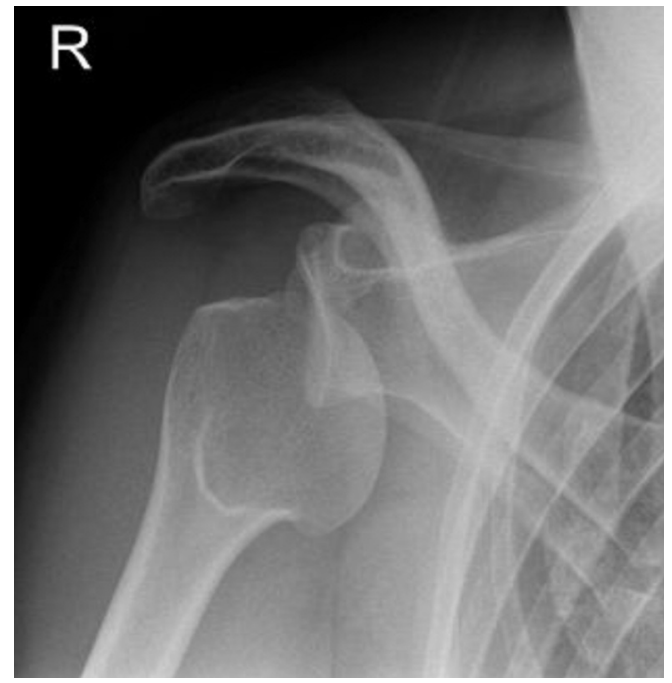

Fig. 1a: Radiograph of shoulder showing anterior shoulder dislocation.

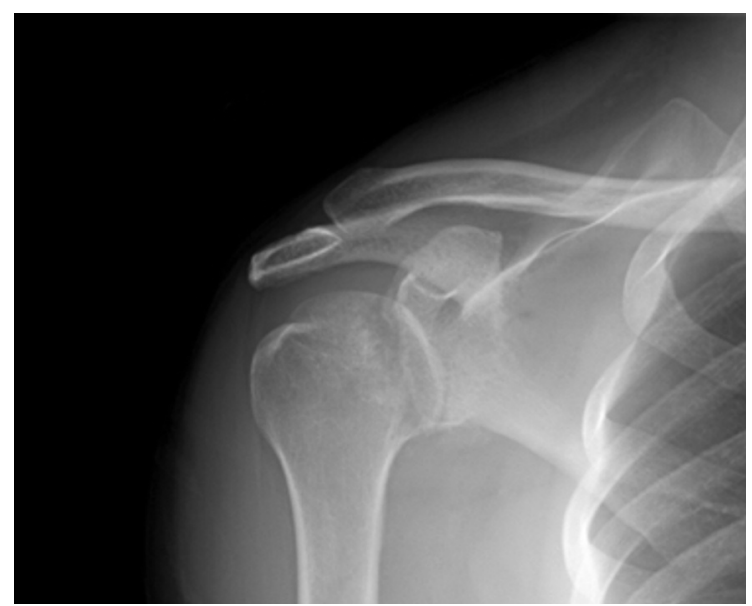

Fig. 2: Radiograph of right shoulder after closed reduction.

influence of alcohol may not complain of pain meaning these injuries may be missed ${ }^{1,3}$. In cases of high velocity trauma injuries, multiple dislocations may occur. For this kind of high energy trauma, adequate exposure of the whole upper extremity, meticulous clinical examination and radiographs of adjacent joints will protect against missing diagnosis of any injury. A complete shoulder examination sometimes may not be possible because of pain and muscle spasms so there should be a low threshold for obtaining radiographs in such patients.

Mechanism of injury in these patients is difficult to define and often the patient is not able to recount the shoulder position during the trauma as well as the direction of the applied force. Force transmitted through the forearm with the elbow flexed, and shoulder externally rotated may be a possible cause of such dislocations ${ }^{4}$. In the present case, the injury to the driver (our patient) was caused by collision with

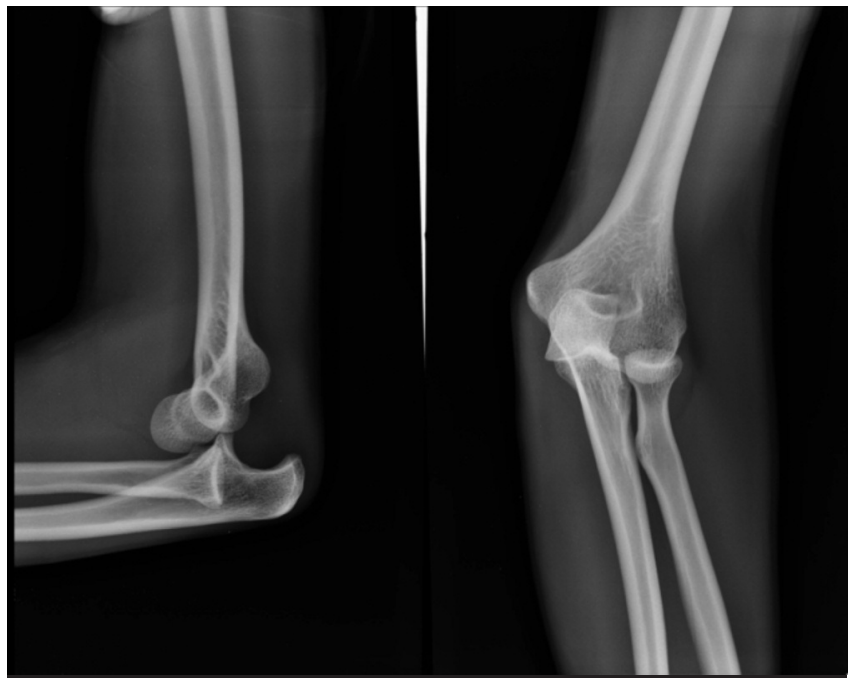

Fig. 1b \& 1c: Anteroposterior and lateral radiograph of the elbow joint showing posterior elbow dislocation.

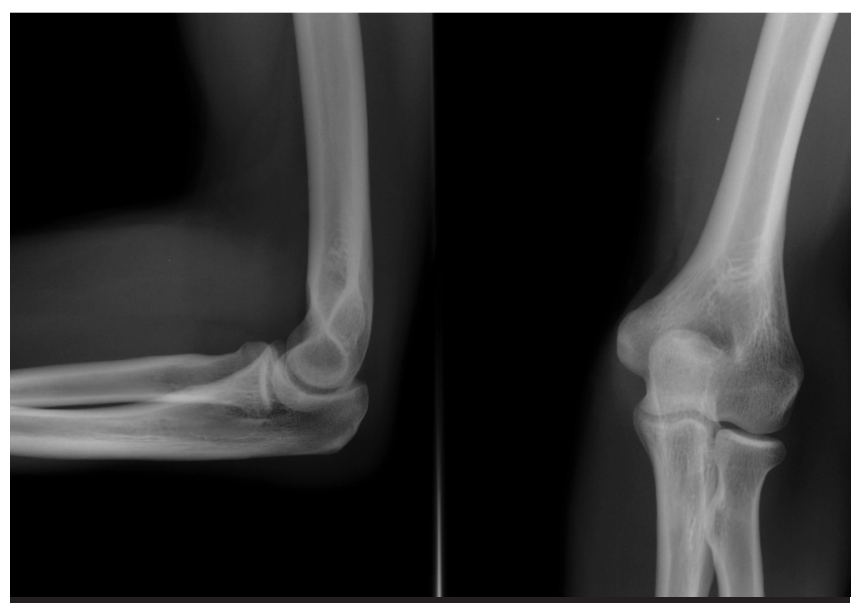

Fig. 2b \& 2c: Anteroposterior and lateral radiograph of elbow after closed reduction.

another vehicle. The frontal impact and protective positioning of the affected limb may have caused such trauma. The elbow dislocation should be reduced first because this will provide a more stable lever arm to reduce the shoulder ${ }^{3,4}$. We found Kocher's method suitable for reduction of the shoulder joint.

\section{CONCLUSION}

Ipsilateral shoulder and elbow dislocation are uncommon and can be treated conservatively with good results. The elbow should be reduced first.

\section{CONFLICT OF INTEREST}

The authors has no conflict of interest with this work. 


\section{REFERENCES}

1. Inan U, Cevik AA and Omeroğlu H. Open humerus shaft fracture with ipsilateral anterior shoulder fracture-dislocation and posterior elbow dislocation: a case report. J Trauma. 2008; 64(5): 18383-6.

2. Suman RK. Simultaneous dislocations of the shoulder and the elbow. Injury. 1981; 12(5): 438.

3. Ali FM, Krishnan S and Farhan MJ. A case of ipsilateral shoulder and elbow dislocation: an easily missed injury. J Accid Emerg Med. 1998; 15(3):198.

4. Khan MR, Mirdad TM. Ipsilateral dislocation of the shoulder and elbow. Saudi Med J. 2001; 12: 1019-21.

5. Ahmet Imerci, Mert Kumbaraci, Mustafa İncesu, Ahmet Savran, Levent Karapinar. Ipsilateral Simultaneous Shoulder and Elbow Dislocation: A Case Report. Tr J Emerg Med. 2011; 11(2):72-5. 